\title{
Influence of Protonation State on the Excited State Dynamics of a Photobiologically Active Ru (II) Dyad
}

\author{
Christian Reichardt, ${ }^{\mathrm{a}, \mathrm{b}}$ Tariq Sainuddin, ${ }^{\mathrm{c}}$ Maria Wächtler, ${ }^{\mathrm{b}}$ Susan Monro, ${ }^{\mathrm{c}}$ Stephan Kupfer, ${ }^{\mathrm{a}}$ Julien \\ Guthmuller, ${ }^{\mathrm{d}}$ Stefanie S. Gräfe, ${ }^{\mathrm{a}}$ Sherri McFarland, ${ }^{\mathrm{c}, \mathrm{e}}$ and Benjamin Dietzek ${ }^{* a, b}$ \\ ${ }^{a}$ Institute of Physical Chemistry and Abbe Center of Photonics, Friedrich-Schiller-University Jena, Helmholtzweg 4, 07743 , \\ Jena, Germany \\ ${ }^{b}$ Leibniz Institute of Photonic Technology (IPHT), Albert-Einstein-Straße 9, 07745, Jena, Germany \\ ${ }^{c}$ Department of Chemistry, Acadia University, Wolfville, NS B4P 2R6, Canada \\ ${ }^{d}$ Gdansk University of Technology, Narutowicza 11/12, 80-233, Gdansk, Poland \\ ${ }^{e}$ Department of Chemistry \& Biochemistry, University of North Carolina Greensboro, Greensboro, North Carolina 27402, \\ United States
}


Quantum chemical calculations

\section{RuH}
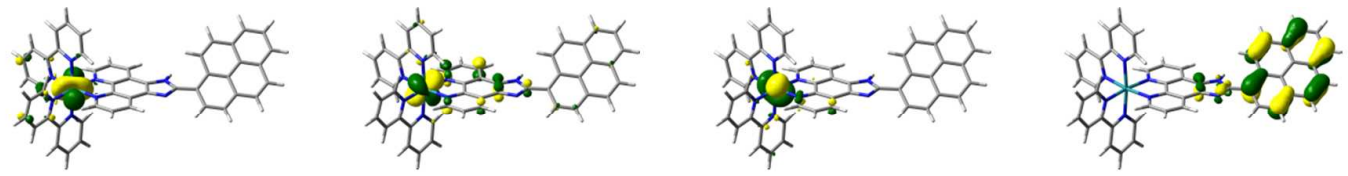

$d_{x y}(195)$

$d_{x z}(196)$

$d_{y z}(197)$

$\pi_{\mathrm{pyr}}(198)$
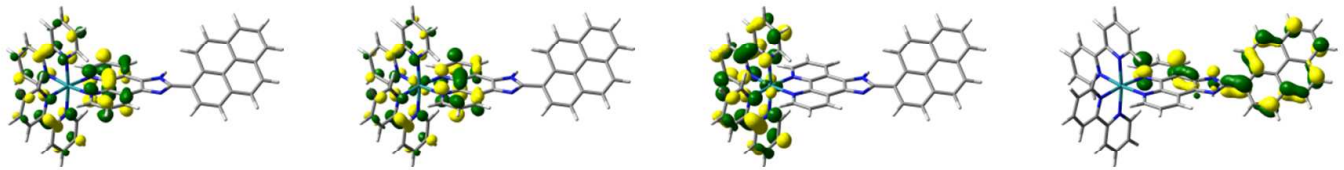

$\pi_{\text {bpy,phen }}(199)$

$\pi_{\text {bpy,phen }}^{*}(200)$

$\pi^{*}{ }_{\text {bpy }}(201)$

$\pi_{\text {phen,im,pyr }}^{*}(202)$

\section{$\mathrm{RuH}_{2}$}
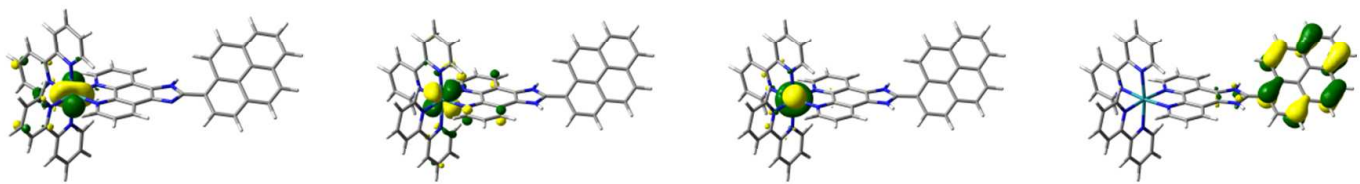

$d_{x y}(195)$

$d_{x z}(196)$

$d_{y z}(197)$

$\pi_{\mathrm{pyr}}(198)$
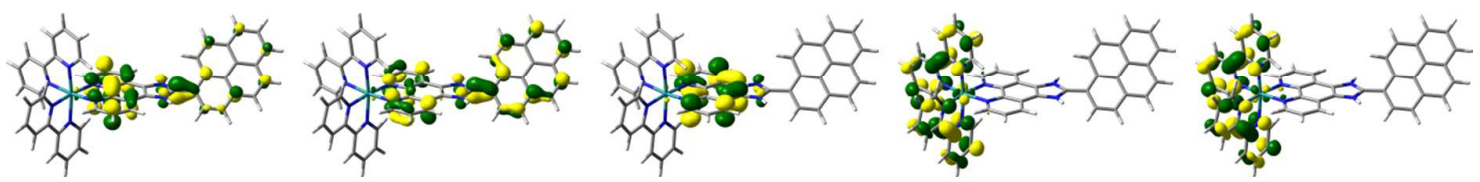

$\pi_{\text {phen }}^{*}(199)$

$\pi_{\text {phen,im,pyr }}^{*}(200)$

$\pi_{\text {phen,im }}^{*}(201)$

$\pi_{\text {bpy }}^{*}(202)$

$\pi_{\text {bpy }}^{*}(203)$

\section{Ru}
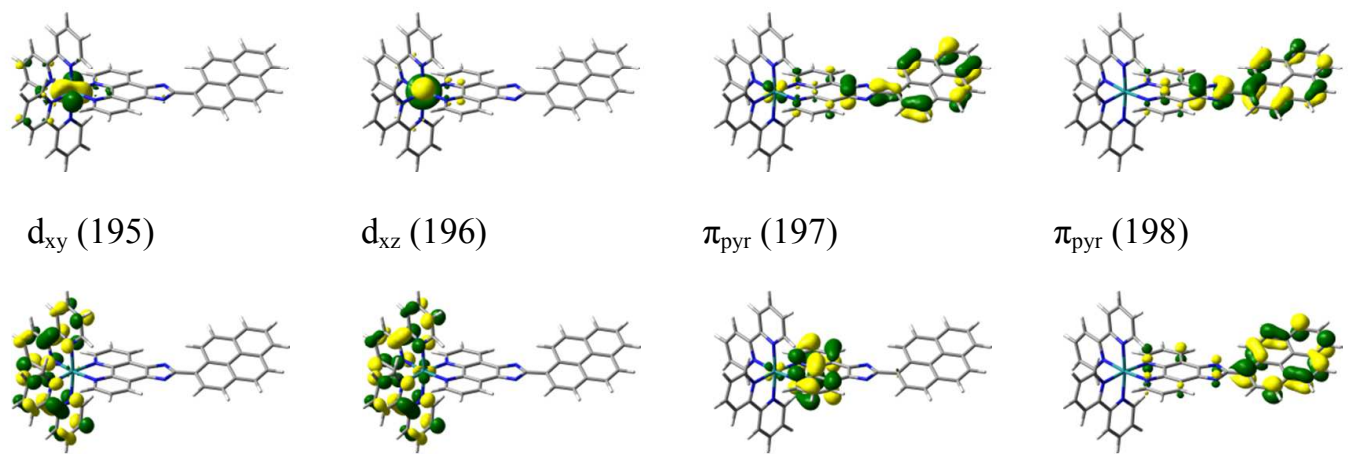

$d_{x z}(196)$

$\pi_{\mathrm{pyr}}(197)$

$\pi_{\mathrm{pyr}}(198)$

$$
\pi_{\text {bpy }}(199)
$$
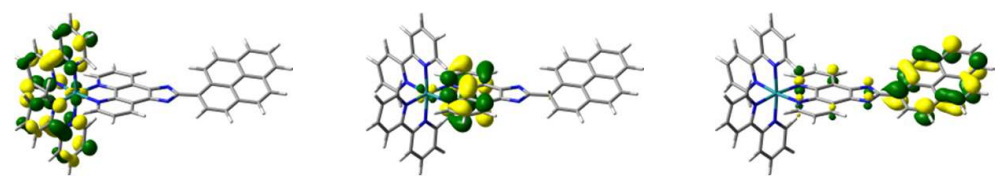

$\pi^{*}{ }_{\text {bpy }}(200)$

$\pi_{\text {phen }}^{*}(201)$

$\pi_{\text {pyr }}^{*}(202)$

Figure S1: Molecular orbitals involved in the main configurations of the electronic states responsible for the absorption properties of $\mathbf{R u H}, \mathbf{R u H}_{\mathbf{2}}$ and $\mathbf{R u}$. The number of the molecule orbital is displayed in parentheses. 


\section{$\underline{\text { Resonance Raman spectroscopy }}$}

The resonance Raman (RR) spectra have been simulated within the independent mode displaced harmonic oscillator model (IMDHOM) assuming that the electronic ground and excited state potentials are harmonic and merely displaced and, thus, share the same set of vibrational modes. Detailed information with respect to the computational methods employed were described previously ${ }^{1}$.

The calculation of the RR intensities of $\mathbf{R u}, \mathbf{R u H}$ and $\mathbf{R u H}_{\mathbf{2}}$ was performed using excitation at $458 \mathrm{~nm}$, while the experimental broadening was reproduced by a dumping factor of $\Gamma=1500 \mathrm{~cm}^{-1}$ describing homogeneous broadening. The RR spectra were obtained using the B3LYP, B3LYP35 and the CAM-B3LYP functionals, but only the data from the latter is presented.

For $\mathbf{R u}$ contributions to the excited states, $\mathrm{S}_{1}, \mathrm{~S}_{6}$ and $\mathrm{S}_{7}$ have been taken into account, while the excitation energies of the pure intra-ligand charge transfer states (ILCT), $\mathrm{S}_{1}$ and $\mathrm{S}_{6}$, were slightly red-shifted by $1000 \mathrm{~cm}^{-1}$ and of the $S_{7}$ (MLCT state) red-shifted by $6500 \mathrm{~cm}^{-1}$. In the case of the neutral form RuH, the $\mathrm{S}_{4}, \mathrm{~S}_{7}$ and $\mathrm{S}_{9}$ contribute to a (partial) resonance at the given excitation wavelength. The MLCT states $\mathrm{S}_{4}$ and $\mathrm{S}_{7}$ were shifted bathochromically by $5000 \mathrm{~cm}^{-1}$, and the ILCT state $\left(\mathrm{S}_{9}\right)$ by $2500 \mathrm{~cm}^{-1}$. The doubly protonated $\mathbf{R u H}_{2}$ has contributions from MLCT states $\mathrm{S}_{4}$ and $\mathrm{S}_{7}$ as well as the ILCT state $S_{9}$ to the RR intensity pattern, while shifts of -5000 and $-2500 \mathrm{~cm}^{-1}$ were applied to the excitation energies of the MLCT and ILCT states, respectively.

CAM-B3LYP is known to overestimate the excitation energy of MLCT states, while the position of ILCT states is much more reliably predicted. The shift decrease for the MLCT states (from 6500 to $5000 \mathrm{~cm}^{-1}$ ) and increase for the ILCT states (from 1000 to $2500 \mathrm{~cm}^{-1}$ ) from $\mathbf{R u}$ to $\mathbf{R u H} / \mathbf{R u H}_{2}$ is deduced from the fact that the ILCT character rises with protonation for the MLCT states and the MLCT character of the ILCT states also increases. Consequently, the trend of mixing in the electronic character is considered for the applied shifts as well.

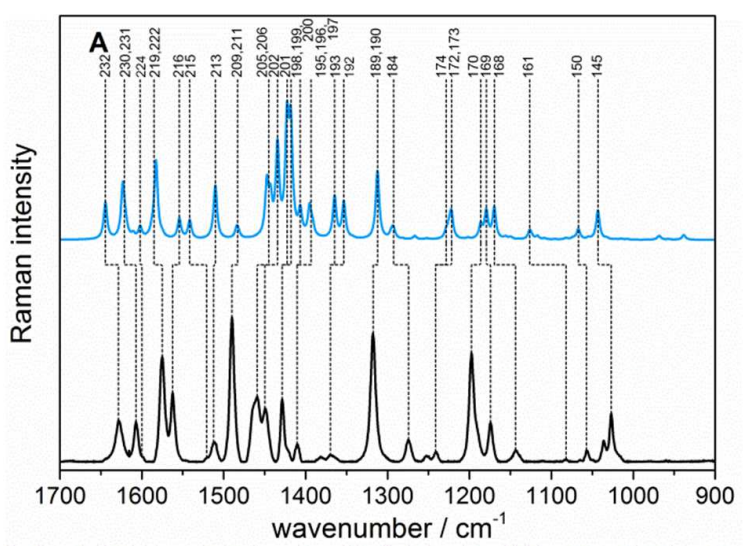



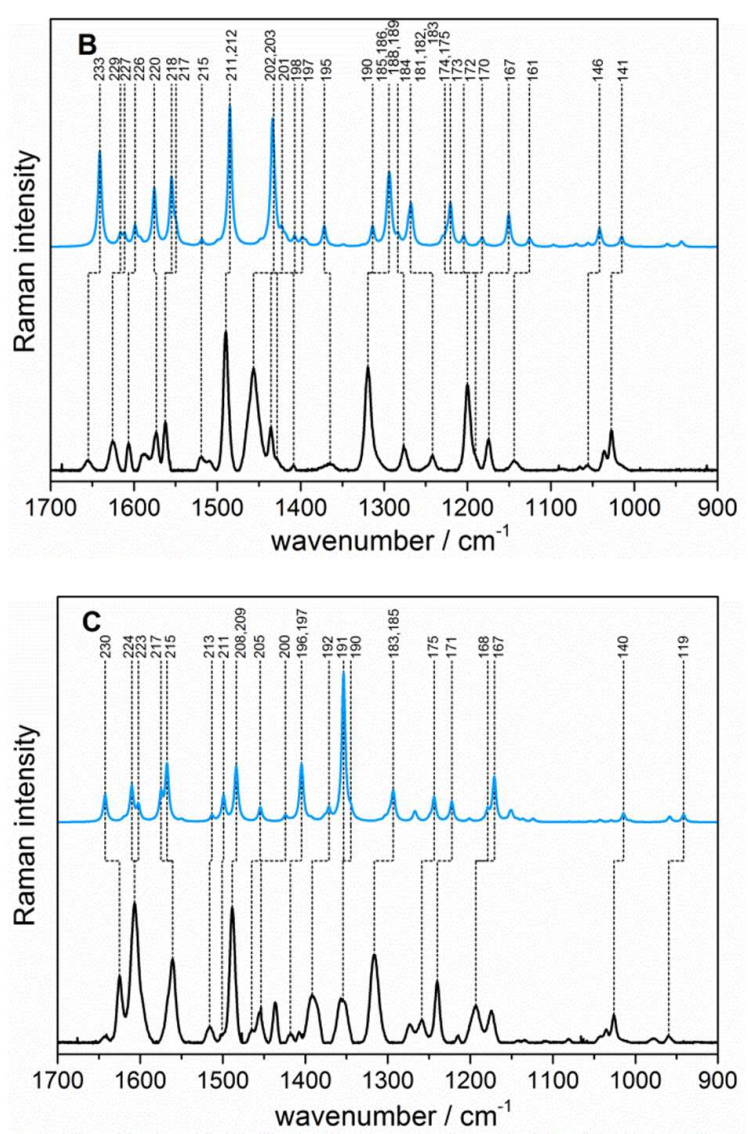

Figure S2: Comparison between experimental (black) and calculated (blue) resonance Raman spectra $\left(\lambda_{\mathrm{ex}}=458 \mathrm{~nm}\right)$ of $(\mathrm{A})$ $\mathbf{R u H}$, (B) $\mathbf{R u H}_{2}$, (C) Ru. 


\section{Femtosecond transient absorption spectra of $\mathbf{R u}$}
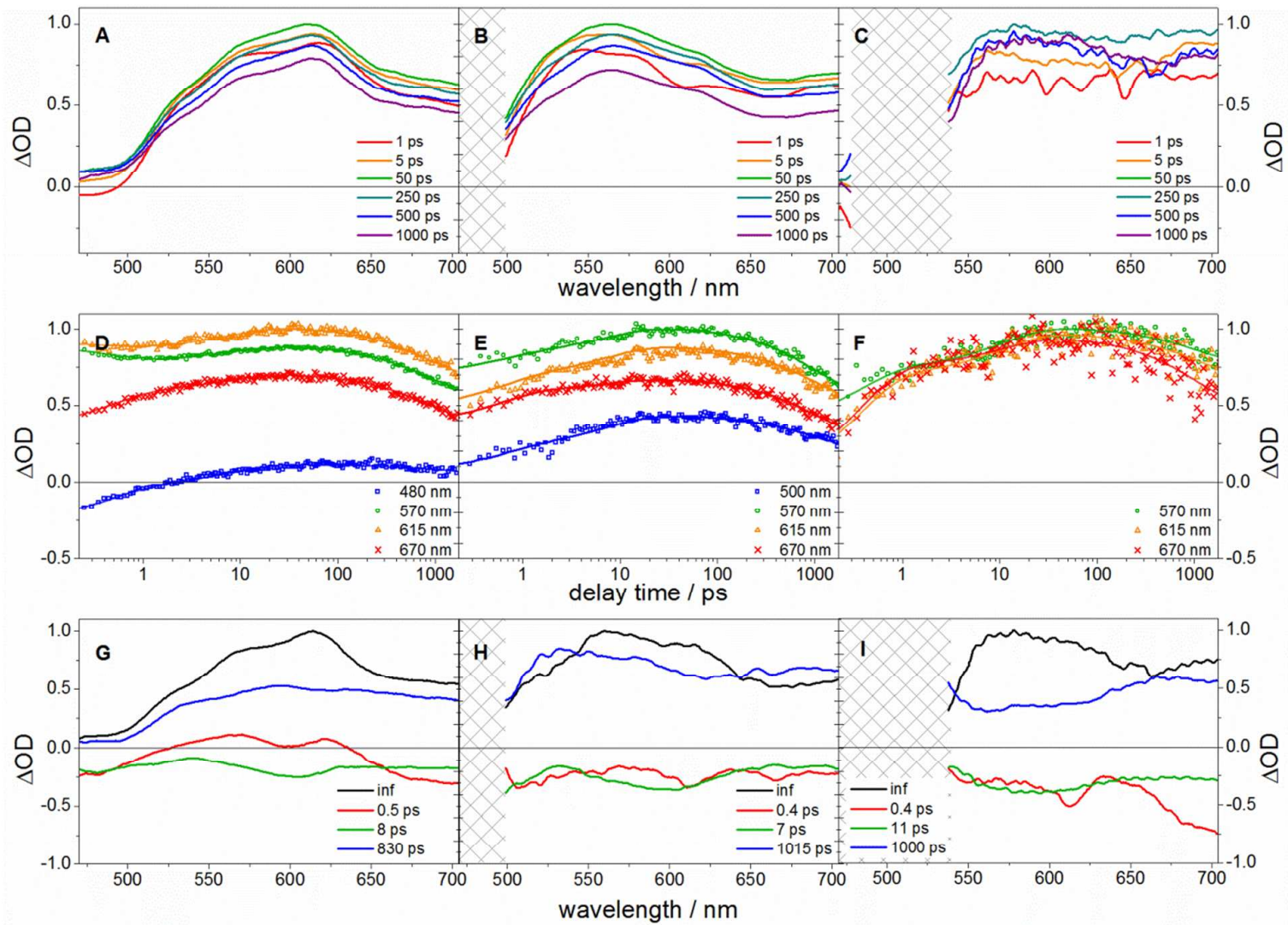

Figure S3: Transient absorption spectra at different delay times (A-C), transient absorption kinetics at selected wavelengths (D-F), and decay-associated spectra and values of time-constants for Ru (G-I) upon pumping at (A/D/G) $403 \mathrm{~nm},(\mathrm{~B} / \mathrm{E} / \mathrm{H})$ $483 \mathrm{~nm}$, or (C/F/I) $513 \mathrm{~nm}$. All spectra are normalized to the maximum value of the data. Scattering of the pump beam in the spectra (B)-(C), (H)-(I) has been omitted for clarity.

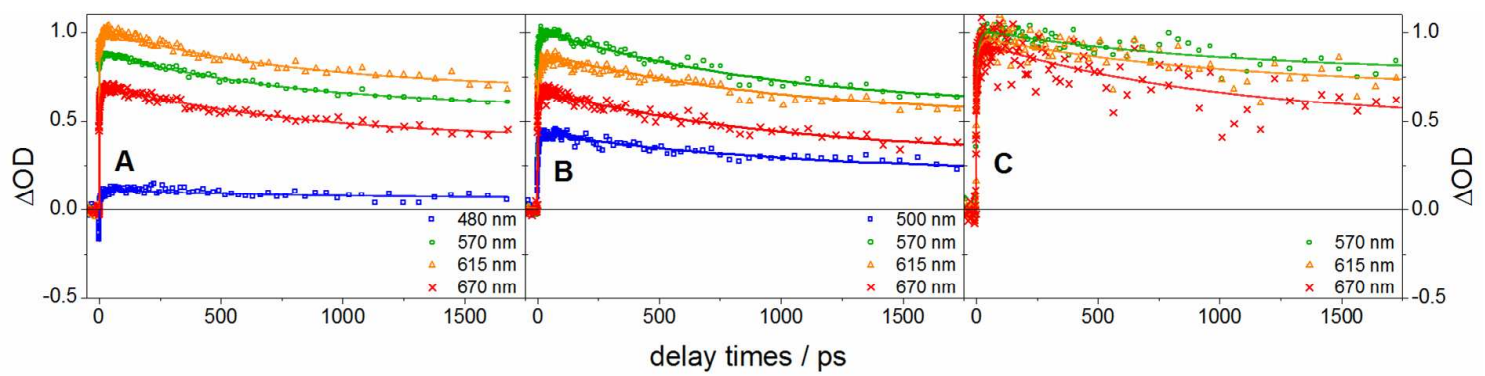

Figure S4: Linear plotted transient absorption kinetics of Ru measured at the selected wavelength upon pumping at (A) $403 \mathrm{~nm}$, (B) $483 \mathrm{~nm}$, or (C) $513 \mathrm{~nm}$. 
$\underline{\text { Nanosecond transient absorption for } \mathbf{R u H}_{2}} \underline{\text { and } \mathbf{R u}}$
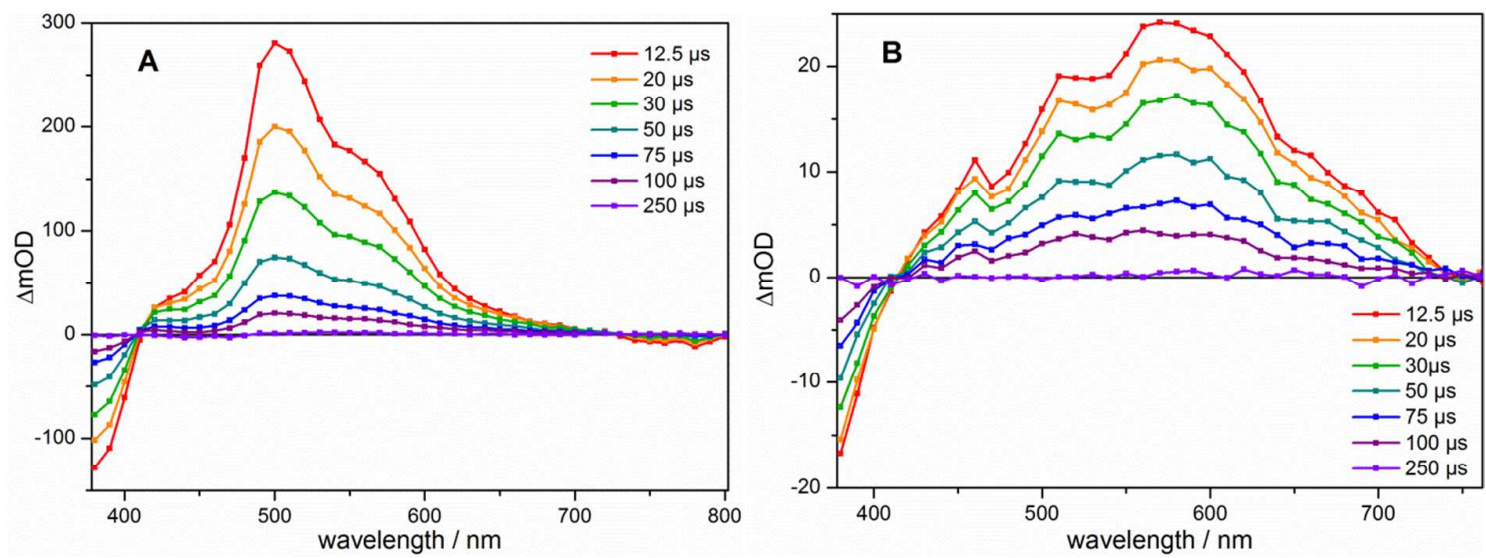

Figure S5: Nanosecond transient absorption spectra recorded for (A) $\mathbf{R u H}_{\mathbf{2}}$ and (B) $\mathbf{R u}$ in water at different delay times upon pumping at $410 \mathrm{~nm}$.

(1) Wächtler, M.; Guthmuller, J.; González, L.; Dietzek, B. Analysis and Characterization of Coordination Compounds by Resonance Raman Spectroscopy. Coord. Chem. Rev. 2012, 256 (15-16), 1479-1508. 